\title{
Relapsing Polychondritis with Chronic lymphocytic Leukemia: Case Report
}

\author{
Barham IA ${ }^{1 *}$, Wahdan $\mathrm{AAM}^{2}$, Ali SM${ }^{1}$ and Alaqraa \\ AS $^{1}$ \\ ${ }^{1}$ Department of Medicine, Al-Quds University, Qalqilia, \\ West Bank, Palestine \\ ${ }^{2}$ Department of Internal Medicine, Palestine Medical \\ Complex, Ramallah, West Bank, Palestine \\ *Corresponding author: Ibrahim A Barham, \\ Department of Medicine, Al-Quds University, Qalqilia, \\ West Bank, Occupied Palestinian Territory, Palestine \\ Received: J une 14, 2021; Accepted: J uly 07, 2021; \\ Published: July 14, 2021
}

\author{
Abstract \\ Background: Relapsing polychondritis is an autoimmune rheumatic \\ disease which is associated with malignant diseases, rarely CLL. \\ Case Report: We report a case of a patient who presented with a new \\ diagnosis of both relapsing polychondritis RP and chronic lymphocytic leukaemia \\ (CLL). The patient is a 59-year-old woman who presented with : joint pain, skin \\ discoloration, Swollen joints, and nose. \\ Conclusion: Patients with RP should go further investigations to exclude \\ malignant diseases. \\ Objective: Rare disease \\ Keywords: Polychondritis; Relapsing, Leukemia, Lymphocytic, Chronic, \\ Ear cartilage
}

\section{Background}

Relapsing polychondritis is a rare autoimmune rheumatic disorder characterized by a severe, recurrent episode of inflammation involving cartilaginous structures, predominantly those of the ears , nose, hyaline cartilage of peripheral joints, and laryngotracheobronchial tree. Other affected structures include the eyes, skin, middle and inner ear, cardiovascular system, and central nervous system [1].

Chronic Lymphocytic Leukemia (CLL) is a slowly progressive disorder of abnormal and malignant neoplastic proliferation of $\mathrm{B}$ lymphocytes [2].

Some case reports and articles presumed a link between malignancy and relapsing polychondritis. The most frequently described neoplasms have been hematological disorders, especially myelodysplastic syndromes [3].

\section{Case Presentation}

A 59 year-old-female housewife came to a private clinic with an exacerbation of vomiting in the last 24 hours. The patient is a known case of chronic vomiting and attacks of dyspnea for 10 years. During history taking, she added nose, ears, and chest wall pain. By examination, there was a swollen nose, ears with redness and severe tenderness, Raynaud's phenomenon with proximal interphalangeal joints and metacarpophalangeal joints tenderness. cherry spots on the hard palate.

The patient's vital signs at the visit were as follows: pulse rate, 80 Beats Per Minute (BPM); blood pressure, 120/80 mmHg. Results of previous blood testing were as follows: Hemoglobin (HGB), 13.3 $\mathrm{g} / \mathrm{dL}$; platelets, $214,000 / \mathrm{mm}^{3}$; white blood cell count, $5400 / \mathrm{mm}^{3}$; C-Reactive Protein (CRP) Alanine Transaminase (ALT), 18 unit/L; The international normalized ratio (INR), 0.98. The patient refused hospital admission.
Clinically, she was suspected as a case of relapsing polychondritis. Ear pinna biopsy confirmed the diagnosis; antinuclear antibody test and extractable nuclear antigen panel were negative. Other investigations were as follows: Erythrocyte Sedimentation Rate (ESR), $2 \mathrm{~mm} / \mathrm{hr}$; C-Reactive Protein (CRP), less than $6 \mathrm{mg} / \mathrm{l}$; fasting blood sugar, $101 \mathrm{mg} / \mathrm{dl}$; abdominal ultrasound, small left kidney with $3 \mathrm{~cm}$ cyst, normal liver kidneys, and gallbladder; oesophago-gastroduodenoscopy, esophagitis, mild chronic gastritis. She was treated with leflunomide $20 \mathrm{mg}$ daily; prednisolone $20 \mathrm{mg}$ daily; Sulpiride 50mg daily; Amitriptyline 10mg daily.

After 2 months she returned with an increase in vomiting frequency and headache in the past few days. During the physical examination, there was diffuse musculoskeletal tenderness, faint chest wheezes, and epigastric tenderness. The plan was to give her Prednisolone 20/10 mg alternative day; Venlafaxine $75 \mathrm{mg}$ and added Eletriptan $40 \mathrm{mg}$.

6 months later, she returned with a flare of diffuse musculoskeletal pain including the nose and ears. She also added respiratory symptoms including mild cough, chest pain, and hoarseness of voice. Vital signs were: pulse rate, 78 Beats Per Minute (BPM); blood pressure, 120/78 mmHg; oxygen saturation 98\%. Physical examination showed diffuse joint line tenderness. Fingers with sausage-like appearance, tender costochondral joints, bronchoalveolar breath sounds with wheezes, regular heartbeat $\mathrm{S} 1+\mathrm{S} 2+0$, no murmurs or added sounds, soft abdomen, no lower limbs edema.

After 2 months, Abdominal and pelvic Computed Tomography (CT) with contrast was done and showed Lymphadenopathy with enlarged pelvic and abdominal retroperitoneal lymph nodes and mesenteric lymph nodes.

After 20 days she was referred to an oncology consult who noticed palpable inguinal lymph nodes. PET/CT scan was done before biopsy taking which revealed multiple active nodes above and below the diaphragm measuring less than $3 \mathrm{~cm}$. Peripheral blood flow 
Table 1: Malignant diseases associated with relapsing polychondritis.

\begin{tabular}{|c|c|c|l|l|}
\hline \multicolumn{1}{|c|}{ Case } & Year & Number & \multicolumn{1}{|c|}{ Age of PCA onset (years) } & \multicolumn{1}{|c|}{ Site of malignancy } \\
\hline Michet CJ, et al. [9] & 1986 & 9 & $\begin{array}{l}\text { Colic adenocarcinoma } \\
\text { Myelodysplastic syndrome } \\
\text { Acute myelogenous leukemia } \\
\text { Chronic myelogenous leukemia } \\
\text { Myeloproliferative disorder } \\
\text { Breast cancer } \\
\text { Lung cancer }\end{array}$ & Myelodysplastic syndrome \\
\hline Frances C, et al. [10] & 2001 & 22 & $\begin{array}{l}18 \mathrm{M} / 4 \mathrm{~F} \\
\text { mean } \\
\text { age:63 }\end{array}$ & $\begin{array}{l}\text { Sweet syndrome, } \\
\text { vasculitis, } \\
\text { thrombosis, } \\
\text { panniculitis, } \\
\text { dermatomyositis } \\
* \text { Urticarial papules } \\
\text { IM/1F } \\
\text { Mean age: } \\
61\end{array}$ \\
\hline Washio K, et al. [11] & 2011 & 1 & $55 / \mathrm{M}$ & Myelodysplastic syndrome \\
\hline
\end{tabular}

cytometry revealed lambda restricted monoclonal B Cell lymphocytes that were $\mathrm{CD} 5$ positive, $\mathrm{CD} 23$ positive, and $\mathrm{CD} 38$ negative consistent with CLL/SLL. One month after, an ultrasound breast biopsy (from Rt axillary L.N) report was issued and showed CLL/SLL. She started treatment with rituximab.

\section{Discussion}

$\mathrm{RP}$ is a rare recurrent inflammatory disease that affects cartilaginous tissue, especially the ear, nose, and tracheobronchial cartilage $[4,5]$.

Our patient, having recurrent bilateral auricular chondritis, non-erosive seronegative inflammatory arthritis, nasal chondritis fulfilled the diagnosis criteria of McAdem et al [4] in the absence of evidence of another disease. Many associated autoimmune diseases, connective tissue diseases, vasculitis, and hematologic disorders have been described in one-third of patients [4]. Less frequently, RP has been associated with solid neoplasms [6].

Interestingly, the case reported here is the second to show a possible association between RP and Chronic Lymphocytic Leukemia (CLL) [7]. Chronic Lymphocytic Leukemia (CLL) is the commonest leukemia in western countries. The disease typically occurs in elderly patients and has a highly variable clinical course. Leukemic transformation is initiated by specific genomic alterations that impair apoptosis of clonal B-cells. The diagnosis is established by blood counts, blood smears, and immunophenotyping of circulating Blymphocytes, which identify a clonal B-cell population carrying the CD5 antigen, as well as typical B-cell markers [8].

It is interesting that RP has a significant association with malignancy. Table 1 summarizes example cases of malignancy associated with RP in patients previously reported in the literature. Most instances occur in men at the age of 30-year-old [26-79 years] (Table 1). Most cases concluded that there is an increased relative risk of malignancy in RP with old age, like our patient. Miller et al. [12] described the first case of malignancy presenting as relapsing polychondritis in 1974, and there have been several reports of malignancies presenting as RP since then. RP has a strong association with leukemia and lymphomas in particular.

The patient in this case presented with a CT scan of the abdomen and pelvis dated February 19, 2020, showing numerous enlarged mesenteric lymph nodes measuring up to $0.9 \mathrm{~cm}$. Enlarged periportal lymph nodes measuring up to $1 \mathrm{~cm}$ was noted as well as aortic retroperitoneal lymph nodes measuring up to $1.6 \mathrm{~cm}$ and numerous bilateral inguinal lymph nodes measuring up to $1.2 \mathrm{~cm}$. The liver and spleen were not enlarged She has night sweats but no other B symptoms. On exam she does have bilateral palpable inguinal lymph nodes. Peripheral blood flow cytometry revealed lambda restricted monoclonal B-cell lymphocytes that were CD5 positive, CD23 positive, and CD38 negative consistent with CLL/SLL. PET/CT scan revealed multiple active nodes above and below the diaphragm measuring less than $3 \mathrm{~cm}$. ultrasound-guided core biopsy of the right axillary lymph node revealed CLL/SLL.

The diagnosis of relapsing polychondritis was confirmed in this patient on 4/2019 and was confirmed by histopathology on biopsy from ear pinna cartilage. Moreover, CLL was diagnosed in 3/2020, giving about a 1-year interval between the diagnosis of the two diseases.

We should also mention the poor response of the patient to the treatment of relapsing polychondritis (prednisone + leflunomide), which may suggest that the presence of CLL interferes with the response of the patient to the treatment of relapsing polychondritis. In addition, the improvement in the complaint of this patient after the starting treatment of CLL (rituximab) supports this idea.

\section{Conclusion}

In summary, this case report presents a rare connection between 2 diseases, Relapsing polychondritis and Chronic lymphocytic leukemia. Given the undefined etiology of relapsing polychondritis and the potential connection to CLL, We believe that the difficulty in assessing associations between them is the challenging part of the case report.

We hope this case report will be of benefit to clinicians, in terms of guiding diagnosis and treatment in future similar patients. Providers also must be vigilant in evaluating patients, particularly in cases where RPC is refractory to initial treatment. It is noteworthy to mention that if a patient was given the treatment of relapsing polychondritis (prednisone + leflunomide) and had poor response; it may indicate the presence of CLL (or any other malignancy) as we have seen in our case.

\section{Acknowledgements}

Source(s) of financial support in the form of grants (quote the 
number of the grant) equipment, drugs etc.

\section{References}

1. Borgia F, Giuffrida R, Guarneri F, et al. Relapsing polychondritis: An updated review. Biomed-icines. 2018; 6: 84.

2. Ansari M, Auerbach $M$, Bahrain $H$. A case of CLL that was successfully treated resulted in the immediate development of $A M L$ from a coexisten myeloid line that had been suppressed. Clin Case Rep. 2015; 3: 165-169.

3. Raida BS, Yosra C, Faten F, Mouna S, Moez J, et al. A Relapsing Polychondritis and Malignancies: A Case Report and Review of Literature. J Dermatol Res Ther. 2016; 2: 41.

4. Sosada B, Loza K, Bialo-Wojcicka E. Relapsing polychondritis. Case Rep. Dermatol. Med. 2014; 2014: 791951.

5. Puéchal X, Terrier B, Mouthon L, et al. Relapsing polychondritis. Joint Bone Spine. 2014; 81: 118-124.

6. Granuloma annulare, relapsing polychondritis, sarcoidosis, and systemic lupus erythematosus: conditions whose dermatologic manifestations may occur as hematologic malignancy-associated mucocutaneous paraneoplastic syndromes. Cohen PR. Int J Dermatol. 2006; 45: 70-80.
7. Bochtler $\mathrm{T}$, Hensel M, Lorenz H-M, Ho A-D, Mahlknecht U. Chronic lymphocytic leukaemia and concomitant relapsing polychondritis: a report on one treatment for the combined manifestation of two diseases, Rheumatology. 2005; 44: 1199

8. Hallek M. Chronic lymphocytic leukemia: 2020 update on diagnosis, risk stratification and treatment. Am J Hematol. 2019; 94: 1266-1287.

9. Michet $\mathrm{CJ}$, McKenna $\mathrm{CH}$, Luthra HS, et al. Relapsing polychondritis. Survival and predictive role of early disease manifestations. Ann Intern Med. 1986; 104: 74-78.

10. Frances C, El Rassi R, Laporte JL, et al. Dermatologic manifestations of relapsing polychondritis: A study of 200 cases at a single center. Medicine (Baltimore). 2001; 80: 173-179.

11. Washio K, Oka M, Ohno K, Shimizu H, Kawano S, Kunisada M, et al. Case of recurrent Sweet's syndrome in a patient with relapsing polychondritis and myelodysplastic syndrome. J Dermatol. 2012; 39: 731-733.

12. Audouin J, Le Tourneau A, Molina T, Camilleri-Broet S, Adida C, Comperat $\mathrm{E}$, et al. Patterns of bone marrow involvement in 58 patients presenting primary splenic marginal zone lymphoma with or without circulating villous lymphocytes. British Journal of Haematology. 2003; 122: 404-412. 